\title{
FENOLOGÍA DEL GUAMO Inga edulis (FABALES: MIMOSOIDEAE) EN DOS AGROECOSISTEMAS DEL QUINDÍO, COLOMBIA.
}

\author{
PHENOLOGY OF THE ROPE WHITEY Inga edulis (FABALES: MIMOSOIDEAE) IN TWO \\ AGROECOSYSTEMS OF QUINDÍO, COLOMBIA.
}

Oscar Humberto Marín-Gómez ${ }^{1}$, Andres Felipe Castaño González¹, Germán Darío Gómez Marín²

\author{
${ }^{1}$ Programa de Biología, Universidad del Quindío. oschumar@gmail.com, \\ Felipecastano@uniquindio.edu.co \\ ${ }^{2}$ Reserva El Ocaso, Centro de Estudios e Investigaciones en Biodiversidad y Biotecnología CIBUQ. \\ Universidad del Quindío, germandario@uniquindio.edu.co.
}

\begin{abstract}
RESUMEN
Inga edulis es una especie ampliamente utilizada como sombrío en la zona cafetera de Colombia. En esta investigación se estudió la fenología de esta especie en un agroecosistema ganadero y otro cafetero del departamento del Quindío entre julio de 2005 y junio de 2006. Se realizaron observaciones mensuales de las fenofases vegetativa y reproductiva, se marcaron flores con el fin de observar la duración de los eventos reproductivos y describir el proceso de antésis. Inga edulis presentó todas las fenofases reproductivas y vegetativas, aunque la caída de follaje no fue evidente. En ambos agroecosistemas los árboles presentaron dos periodos de floración con una duración de 4 a 6 meses, mientras que la fructificación, la brotación y la caída del follaje fueron constantes. La intensidad de algunas fenofases mostró una coincidencia con la precipitación. Nuestros resultados muestran diferencias en el periodo de antésis y sincronía de las fenofases con respecto a otros estudios en el Neotrópico, lo que sugiere una posible variación fenológica a lo largo de su distribución geográfica.
\end{abstract}

Palabras claves: Inga edulis, fenología, antésis, producción de frutos, agroecosistemas, Quindío.

\begin{abstract}
Inga edulis is a widely used shadow species in the coffee plantations of Colombia. The phenology of this species was studied in a silvopastoril and coffee agroecosystem in the Quindío, department, Colombia. Based on monthly observations, the vegetative and reproductive phases were recorded. Additionally, the flowers were marked with the aim to observe the duration of the reproductive events and to describe the anthesis. Inga edulis showed all the reproductive and vegetative phenophases, though the foliage fall was not evident. In both agroecosystems, the plants suffered two periods of flowering, each with 4 to 6 months of duration, whereas fruiting, leaf production and foliage fall were constant. The intensity of some phenophases showed a coincidence with the precipitation. There are marked differences in the period of anthesis phenology synchronization between our results and similar studies in the tropics, which suggests a possible phenological variation along their geographical distribution.
\end{abstract}

Key words: Inga edulis, phenology, anthesis, fruit production, agroecosystems, Quindío. 


\section{INTRODUCCIÓN}

El género Inga es uno de los grupos más complejos y diversos de la flora neotropical con cerca de 300 especies y un importante centro de especiación en la Amazonía (1,2 y 3). Los grandes despliegues florales que exhiben la mayoría de las especies y el mutualismo con hormigas y avispas parasitoides que visitan los nectarios extraflorales, constituyen las características más llamativas de este género $(4,2)$.

Inga edulis es conocido comúnmente como inga cipo, pacay, pacae, catetajo, guamo o guama. Su distribución natural comprende el este de los Andes de Colombia hasta el norte de Argentina y la costa Atlántica de Brasil entre los $750 \mathrm{~m}$ y los $1600 \mathrm{~m}$ (2), es originario de la Amazonía y ha sido introducido por el hombre en varias regiones del Neotrópico como árbol de sombra en plantaciones de café y cacao $(5,2)$. Es una especie de rápido crecimiento ampliamente utilizada como sombrío en la zona cafetera colombiana por los múltiples beneficios que brinda a la productividad del café (6). Además, presenta una floración masiva que atrae a una gran variedad de visitantes florales como artrópodos y aves $(7,8)$.

La mayoría de las investigaciones sobre la ecología del género Inga han sido realizadas principalmente en Centroamérica, en donde se ha estudiado la biología floral, la fenología, la polinización, las interacciones con hormigas y la importancia para las comunidades de aves e insectos $(4,9,10$ y 11). Los pocos estudios fenológicos sobre el género Inga son los de Koptur (4,21 y 25), en Costa Rica y el de Falcão y Clement (12) en Brasil. Otros autores como Feinsinger et al. (1985) en Trinidad y Tobago, Custodio \& Morellato (2000) en el sureste de Brasil, incluyeron en sus investigaciones a I. edulis pero no presentan datos detallados sobre sus patrones fenológicos. Para I. edulis no se tienen datos sobre su fenología en los Andes de Colombia, por lo tanto los objetivos de este trabajo fueron describir las fases vegetativas y reproductivas de I. edulis y comparar la distribución de estas fenofases entre un agroecosistema ganadero y uno cafetero entre el departamento del Quindío, Colombia.

\section{MATERIALES Y METODOS}

Este estudio se realizó en dos agroecosistemas en el departamento del Quindío, Colombia. El agroecosistema ganadero se encuentra ubicado en la Reserva Natural "La Montaña del Ocaso" (04³4'N; 075 $51^{\circ}$ 'W), municipio de Quimbaya, a $1100 \mathrm{~m}$ de altitud, con una pre- cipitación promedio anual de $1691 \mathrm{~mm}$ y una temperatura de $24^{\circ} \mathrm{C}(18)$. La vegetación esta compuesta de pequeños parches de Guadua angustifolia, un fragmento de bosque secundario de 106 ha y algunos potreros arborizados con I. edulis y cercas vivas de matarratón (Gliricidia sepium). En este sitio los árboles de I. edulis presentan una distribución agregada. El agroecosistema cafetero se encuentra ubicado en el municipio de Calarcá a una altura de 1500 m.s.n.m en la Vereda "La Zulia" $\left(04^{\circ} 32^{\prime} \mathrm{N}\right.$; 75³9' $\left.\mathrm{W}\right)$, el cual presenta una precipitación promedio anual de $3134.6 \mathrm{~mm}$ y una temperatura de $20^{\circ} \mathrm{C}$. La Zulia es una finca cafetera tecnificada de 25 ha, que aún conserva un sombrío disperso compuesto principalmente de árboles de I. edulis, y en menor proporción I. densiflora e Inga sp.

En cada agroecosistema se seleccionaron al azar 15 individuos de I. edulis para hacer un seguimiento de su fenología. Las observaciones se realizaron utilizando binoculares 10x42 cada 15 días entre Julio de 2005 a Junio de 2006. Durante cada visita se contaron el número de botones florales, de flores (jóvenes y maduras) y de frutos (verdes y maduros) en todo el árbol y se estimó el porcentaje de brotación foliar y caída de follaje. La intensidad de las diferentes fenofases se cuantificó siguiendo las 5 categorías de Fournier y Charpentier (15). Adicional a esto, se realizó un seguimiento a 10 inflorescencias en estado de botón floral ( $\mathrm{n}=10$ árboles/sitio) durante 4 meses, para observar la duración de los eventos reproductivos (desde botón floral hasta fruto maduro) y describir el proceso de antésis. Durante estas observaciones se describió el color, tamaño, número de botones florales, flores jóvenes, flores maduras, frutos verdes y frutos maduros.

Se utilizaron pruebas no paramétricas debido al tamaño de la muestra y porque los datos no presentaron distribución normal ni homogeneidad de varianzas. La prueba de la U de Mann-Whitney se utilizó para identificar si existían diferencias significativas en la producción de frutos. Estos análisis se realizaron utilizando el programa STATISTICA 5.1 (17).

\section{RESULTADOS}

La caída de follaje no fue evidente en I. edulis, mientras que la brotación de follaje fue frecuente en los dos agroecosistemas. Una hoja completó su desarrollo entre 45 y 60 días. La floración comprendió tres estados: botón floral, flor joven y flor madura. El número de botones florales por inflorescencia varió entre 6 y 28 
(promedio $=16$, desviación estándar $=4.37, \mathrm{n}=200$ ), el desarrollo de los botones florales fue acrópeto, al inicio se encontraban agrupados y rodeados por brácteas, presentaron un color verde oliva y un tamaño de $5 \mathrm{~mm}$ de largo y $4 \mathrm{~mm}$ de ancho. Los botones florales mostraron un color verde oliva a verde oscuro, un tamaño de 16 $\mathrm{mm}$ x $8 \mathrm{~mm}$ y tardaron entre 21 y 33 días en desarrollarse cuando las brácteas se cayeron. La producción de botones florales fue sincrónica con la producción de hojas; no todos los botones alcanzaron el estado de flor joven, pues muchos se desprendieron del racimo.

Las flores jóvenes eran ovaladas con el cáliz de color verde oliva a verde oscuro, presentaron un tamaño de $15 \mathrm{~mm}$ x $8 \mathrm{~mm}$ hasta $27 \mathrm{~mm}$ x $9 \mathrm{~mm}$, con los primordios de la corola de color verde claro. El número de flores jóvenes por racimo varió entre 3 y 15 , pero generalmente se observaron entre 4 y 6 en diferentes estados de desarrollo. La fase de flor joven duró entre 5 y 8 días, y continúo con la fase de flor madura. Las flores adultas eran grandes con una longitud entre $78 \mathrm{~mm}$ y 84 $\mathrm{mm}$, con el cáliz tubular pentalobulado de color verde oscuro, la corola gamopétala de color blanco, de forma tubular, con numerosos estambres blancos (entre $67 \mathrm{y}$ 95) fusionados en su base formando un tubo en el que se acumuló el néctar; el pistilo sobrepasó generalmente a la longitud de los estambres. Las flores maduras eran sesíles y se encontraron agrupadas en racimos erectos, cada racimo presentó de 1 y 12 flores, aunque generalmente se observaron entre 2 y 4 .

El proceso de antésis tuvo una duración de 24 h, este se inició en la tarde a partir de las 13:00 h hasta abrir por completo a las 19:00 h. En este estado las flores eran de color verde claro a blanco y produjeron pequeñas cantidades de néctar, las cuales aumentaron en la noche. A partir de las 12:00 h del siguiente día las flores comenzaron su periodo de senescencia caracterizado por una descomposición progresiva, luego de 2 días las flores tenían un color café claro y generalmente se desprendieron y cayeron al suelo cuando no fueron fecundadas.

A partir de la fecundación de la flor, el desarrollo del fruto duró entre 50 y 60 días hasta alcanzar su madurez total y caer del árbol. Los frutos se agruparon en infrutescencias y se encontraron entre 3 y 7 , aunque su número decreció con el estado de madurez del fruto. La fase de fruto inmaduro duró entre 20 a 30 días y se inició con la caída de la corola y los estambres; este presentó una coloración café y una longitud de 40 a $150 \mathrm{~mm}$, un ancho entre 5 y $15 \mathrm{~mm}$, con pubescencia a lo largo de este. El fruto maduro era una legumbre tomentosa recta o sigmoidea de color ferrugineo, de una longitud entre 195 y $510 \mathrm{~mm}$ y un ancho de 20 a 30 $\mathrm{mm}$, el peso varió entre 51 y $118 \mathrm{~g}$. Cuando alcanzaron su máximo desarrollo, las vainas experimentaron una dehiscencia longitudinal, debido al crecimiento de la semilla y la presión del arilo. Cada fruto presentó entre 6 y 32 semillas de color morado recubiertas por un arilo de color blanco, suculento y dulce. Las semillas tenían una longitud de 15 y $20 \mathrm{~mm}$, un ancho de 10 y $13 \mathrm{~mm}$, y un peso de entre 0.7 a $0.9 \mathrm{~g}$. Los frutos que se cayeron de los árboles tenían algunas semillas que presentaron indicios de germinación.

Inga edulis presentó una brotación foliar continua a lo largo del periodo de estudio, con picos superiores al $50 \%$ en los meses de noviembre-diciembre (Fig. 1), los cuales coincidieron con la temporada de mayor precipitación en los dos sitios de estudio y fueron de mayor intensidad en el agroecosistema cafetero (Fig. 2). La fase caída de follaje no fue conspicua, dado que no se presentó en un porcentaje alto, para ambos sitios se observaron picos en los meses de abril-junio, siendo mayores en agroecosistema ganadero (Fig. 1).

Inga edulis presentó dos periodos de floración, el primero de junio-diciembre y el segundo de febrero-junio. La duración de esta fase fue de 4 a 5 meses en el agroecosistema ganadero y de 5 a 6 meses en cafetero. Se presentaron dos picos de floración superiores al $50 \%$, entre los meses de septiembre-octubre y marzo-abril, los cuales coincidieron con el incremento de la precipitación en ambos sitios (Figs. 1 y 2). Aunque la fructificación fue constante a lo largo del año, la producción de frutos fue baja con picos en los meses de diciembre, febrero y junio en el agroecosistema cafetero y de marzo y diciembre en el cafetero. En general los picos de fructificación ocurrieron durante la transición entre las épocas de mayor y menor precipitación (Figs. 1 y 2).

La producción mensual y anual de inflorescencias por individuo fue mayor en el agroecosistema ganadero. La floración fue de mayor intensidad en el agroecosistema ganadero durante la mayoría del año, excepto en los meses de agosto y mayo en los que fue mayor en el cafetero, en ambos sitios la producción de flores jóvenes fue superior al número de flores adultas, pero esta diferencia aumentó al finalizar el segundo periodo de floración. La producción de frutos baja con relación al número de flores producidas por árbol. El porcentaje de producción de frutos verdes (FRV) y maduros (FRM) 




Figura 1. Distribución de las fenofases reproductivas y vegetativas de I. edulis en el agroecosistema ganadero (líneas azules) y cafetero (líneas rojas). A: Floración, B: Fructificación, C: Brotación, D: Caída de Follaje. 


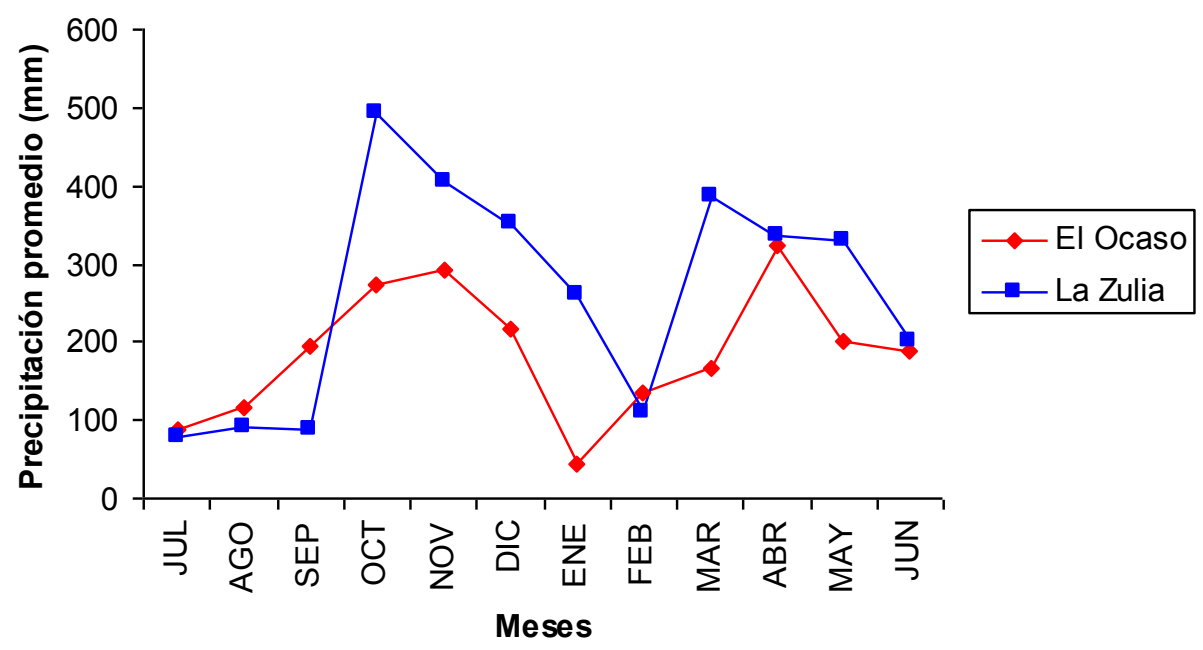

Figura 1. Distribución de la precipitación promedio anual en el área de estudio. Fuente: Estación climática Portátil de la reserva "El Ocaso" CENICAFE Municipio de Quimbaya (2005-2006). Estación climática de la Corporación Autónoma del Quindío, Municipio de Armenia (2005-2006).

no presentó diferencias significativas entre sitios ( $\mathrm{U}$ de Mann Whitney, FRV: $\mathrm{U}=47.5, \mathrm{P}=0.843$; FRM: $\mathrm{U}=$ $46 ; \mathrm{P}=0.731)$. En agroecosistema cafetero la frecuencia de flores que no produjeron frutos y se cayeron del árbol fue mayor que en el ganadero $(89.5 \%$ vs $61 \%$; U de Mann Whitney: $U=5.5, P=0.000695)$. Sin embargo el porcentaje de flores que no produjeron frutos en ambos sitios fue similar (90\%; U de Mann Whitney: U $=49, \mathrm{P}=0.9397)$.

\section{DISCUSIÓN}

La variabilidad fenológica se presenta a nivel individual, intraespecífico, poblacional, comunitario y filogenetico (16). Esta variabilidad puede ser producto de las respuestas particulares de las especies y gremios, o a las diferencias entre individuos según su estado fisiológico y distribución en determinados microhábitats e incluso a la dinámica diferencial de las partes de un individuo (16). Las poblaciones de I. edulis en los agroecosistemas estudiados fueron similares en cuanto a la sincronización de su fenología vegetativa y reproductiva, aunque se encontraron diferencias en la intensidad de algunas fenofases reproductivas (botones florales y frutos verdes), las cuales pueden asociarse a la variabilidad entre individuos y no entre poblaciones. Inga edulis puede considerarse como una especie asincrónica debido a que varios individuos de las dos poblaciones estudiadas mostraron distintos ritmos fenológicos o se encontraban en diferentes fases simultáneamente, un patrón observado en varios bosques del Neotrópico (16, 18 y 22).
En la Amazonía central en Brasil, I. edulis presentó una perdida parcial de hojas durante la fructificación, mientras que la brotación foliar ocurrió al inicio de la floración (12), en el presente estudio se encontró lo contrario, la caída de follaje fue constante a lo largo del año, mientras que la mayor producción de follaje se presentó al finalizar la floración, coincidiendo con la temporada de mayor precipitación. Inga edulis no presentó una caída de follaje evidente, sin embargo la gran cantidad de hojas encontradas en el suelo durante todo el año sugiere que esta fenofase es continua pero difícil de registrar, como ocurre con otras especies (18). El inicio de la floración de I. edulis en el área de estudio ocurrió al igual que otras especies de Inga (21), durante el periodo de transición entre la estación "seca" a "húmeda", pero los picos de floración coincidieron con un incremento de la precipitación. En Costa Rica, la fenología floral de las especies de Inga no fue uniforme y estuvo espaciada a lo largo del año, muchas especies florecieron entre la estación "seca" y la "húmeda" (21), este mismo patrón ha sido observado en varias comunidades de árboles neotropicales (22).

En general los árboles del género Inga presentan floraciones masivas y continuas con varios picos al año (2,21 y 23), el periodo de floración varia en tiempo (entre 2 a 6 meses según la especie) y en intensidad (la cantidad del número de flores fluctúa a lo largo del periodo de floración). Falcão \& Clement (19), en la Amazonía central de Brasil, observaron que $I$. edulis presentó una floración continua a lo largo del año, pero con picos 
durante algunos meses del año. En los agroecosistemas estudiados se observó sincronía de la floración a nivel poblacional y variación a nivel individual en las poblaciones. Sin embargo, a diferencia del estudio de Falcão y Clement (19) I. edulis solo presentó dos picos de floración y su intensidad fue menor que en la Amazonía brasileña. Este patrón de floración constante a lo largo del año ha sido observado en otras mimosáceas $(21,22)$, lo que sugiere que la floración varía a través de su distribución natural, lo que puede estar asociado con el polimorfismo que presenta esta especie $(3,5)$, o con las características del suelo, el fotoperiodo y las variables climáticas de los sitios donde habita.

Las flores de los árboles del género Inga presentan su antésis durante un único periodo del día, o en diferentes cohortes a lo largo del día ya sea en la madrugada, en la mañana, al medio día, o al atardecer (21). Las flores de I. edulis comienzan a abrir en horas de la tarde entre las 13:00-14:00 h, hasta las 18:00-19:00 horas, por lo que su antésis es crepuscular para los árboles estudiados y no ocurre en la madrugada como lo han reportado (12). Al igual que en otros estudios en Costa Rica $(21,23)$ y Brasil (19), la fructificación de I. edulis fue constante entre los meses de agosto y abril, con una intensidad de nueve meses. Durante este periodo se presentaron dos picos de producción de frutos que ocurrieron durante la época de bajas precipitaciones.

Todas las especies del género Inga son autoincompatibles y se caracterizan por tener grandes despliegues florales con una baja producción de frutos $(22,23)$, la baja producción de frutos no se debe a una baja tasa de polinización pues las flores son visitadas por una gran variedad de animales (23). Las flores de I. edulis en el área de estudio son visitadas por varias especies de aves, insectos y dos especies de mamíferos, que actúan como posibles polinizadores o como ladrones de recompensas florales (7). Dentro de este ultimo grupo los coleópteros adultos y las larvas de mariposas y las hormigas arrieras afectan deforma negativa el proceso de polinización pues consumen las flores $(7,8)$.

Las flores de Inga son polinizadas por mariposas diurnas y nocturnas, avispas, abejas, colibríes y murciélagos (2, 21,22 y 23). En la Amazonía brasileña las flores de I. edulis fueron visitadas por abejas, avispas, colibríes y mariposas, siendo las abejas fueron los visitantes mas frecuentes y los principales polinizadores (12). Estos datos de visitantes florales concuerdan con los de nuestro estudio, pero a diferencia de estas investigaciones el número de visitantes y de polinizadores potenciales es superior $(7,8)$. Estos resultados sugieren que I. edulis es una que ofrece una gran variedad de alimento para la fauna local, por lo que debe considerarse como una especie de interés en los proyectos silvopastoriles para el manejo de los agroecosistemas o en la adecuación de corredores biológicos en la zona cafetera.

\section{AGRADECIMIENTOS}

Agradezco a Oscar Enrique Murillo, Andrés Felipe Orozco, Mercedes Girón y Enrique Arbeláez por sus sugerencias, a Rocío García, Ana Lucia López, Carlos Alberto Agudelo y al CIBUQ por su apoyo académico y logístico y a las fundaciones Ideawild y Optics for the Tropics por la donación de equipos. 


\section{BIBLIOGRAFÍA}

1. León, J. Central American and West Indian species of Inga (Leguminosae). Annals of Missouri Botanical Garden 1966; 53(3): 265-359.

2. Pennington, T. D. The genus Inga Botany. The Royal Botanical Gardens. London, England. 1997.

3. Richardson, J. E., R. T. Pennington, T. D. Pennington \& P. M. Hollingsworth. Rapid Diversification of a Species-Rich Genus of Neotropical Rain Forest Trees. Science 2001; 293(5538): 2242-2245

4. Koptur, S. Outcrossing and pollinator limitation of fruit set: breeding systems of Neotropical Inga trees (Fabaceae: Mimosoideae). Evolution 1984; 38: 1130-1143.

5. Sousa, M. S. El género Inga (Leguminosae: Mimosoideae) del sur de México y Centroamérica, estudio previo para la flora Mesoamericana. Annals of Missouri Botanical Garden 1993; 80: 223-269.

6. Cardona-Calle D. A., \& S. Sadeguian. Beneficios del sombrío de guamo en suelos cafeteros. Avances Técnicos Cenicafé No. 335, 2005.

7. Marín-Gómez, O. H. Composición, actividad de forrajeo y "polinización" de las aves e insectos que visitan el guamo Inga edulis Martius (Fabales: Mimosaceae) en dos agroecositemas del Quindío. Tesis de pregrado. Programa de Licenciatura en Biología y Educación Ambiental. Universidad del Quindío. 2006; 112 pp.

8. Marín-Gómez, O. H. \& R. C. García. Artropofauna asociada al guamo Inga edulis (Fabales: Mimosaceae) en un agroecosistema ganadero del Quindío, Colombia. Rev. Asoc. Col. Cienc. Biol. 2007; 19:49-56

9. Wunderle J. M. \& S. C. Latta. Avian abundance in sun and shade coffee plantations and remnant pine forest in the Cordillera Central, Dominican. The Wilson Ornithological Bulletin 1996; 7: 19-34

10. Calvo, L. \& J. Blake. Bird diversity and abundance on two shade coffee plantation in Guatemala. Bird Conservation International 1998; 8: 297-308

11. Johnson, M. D. Effects of the shade-tree species and crop structure on the winter arthropod and bird communities in a Jamaican shade coffee plantation. Biotropica 2000; 32(1): 133-145

12. Custodio, T. D. \& P. C. Morellato. Fenologia de espécies arbóreas em floresta de planície litorânea do sudeste do Brasil. Revta Brasil. Bot. 2000; 23(1): 13-26

13. Feinsinger, P., L. A. Swarm \& J. A. Wolfe. Nectar-feeding birds on Trinidad and Tobago: Comparison of diverse and depauperate guilds. Ecological Monographs 1985; 55(1): 1-28

14. Agudelo, C. A. \& G. D. Gómez.. Reserva natural La Montaña del Ocaso: un nuevo modelo de conservación. En: Agudelo C A, Vélez M C. Importancia de la microcuenca del río Roble. Monografías de la Flora Andina. Vol. 3. Universidad del Quindío. HUQ. 2001.

15. Fournier, L. A. \& C. Charpantier. El tamaño de la muestra y frecuencia de las observaciones en el estudio de las características fenológicas de los árboles tropicales. Cespedesia 1978; 7: 25-26. Suplemento 2

16. Williams-Linera, G. \& J. Meave. Patrones fenológicos. En: Kattan, G., y M. R. Guariguata. Ecología y Conservación de Bosques Neotropicales. Editorial LUR, Costa Rica. 2002.

17. StatSoft. STATISTICA for Windows. Tulsa, StatSoft, Inc. 1998.

18. Gómez, G. D., C. A. Agudelo, A. F. Orozco, I. G. Mejía, A. Valencia, E. Franco \& D. A. Peña. Fenología de especies forestales. Págs. 252-307 En: Agudelo C A. (Ed.). Riqueza Biótica Quindiana. Centro de Estudios en Biodiversidad y Biotecnología. Universidad del Quindío. Armenia. 2006;421p.

19. Falcão, M. A. \& C. R. Clement. Fenologia e produtividade do ingá cipó (Inga edulis) na Amazônia Central. Acta Amazónica 2000; 30(2):173-180

20. Koptur, S. Flowering phenology and floral biology of Inga (Fabaceae: Mimosoideae). Systematic Botany 1983; 8(4): 354-368

21. Koptur, S. Alternative defenses against herbivores in Inga (Fabaceae: Mimosoideae) over an elevation gradient. Ecology 1985; 66: 1639-1650

22. Frankie, G. W., G. B. Herbert \& P. A. Opler. Comparative phenological Studies of trees in tropical wet and dry forest in the lowlands of Costa Rica. J Ecol. 1974; 62(3): 881-919

23. Villasana, R. A. \& A. Suárez. Estudio fenológico de dieciséis especies forestales presentes en la reserva forestal Imataca Estado Bolivar-Venezuela. Revista Forestal Venezolana 1997; 41(1): 13-21 